\title{
Omentum is highly effective in the management of complex cardiothoracic surgical problems
}

\author{
Joseph B. Shrager, MD* \\ John C. Wain, MD \\ Cameron D. Wright, MD \\ Dean M. Donahue, $\mathrm{MD}^{\mathrm{a}}$ \\ Gus J. Vlahakes, $M D^{\mathrm{b}}$ \\ Ashby C. Moncure, $\mathrm{MD}^{\mathrm{a}}$ \\ Hermes C. Grillo, $\mathrm{MD}^{\mathrm{a}}$ \\ Douglas J. Mathisen, MDa
}

Objectives: Vascularized, pedicled tissue flaps are often used for cardiothoracic surgical problems complicated by factors that adversely affect healing, such as previous irradiation, established infection, or steroid use. We reviewed our experience with use of the omentum in these situations to provide a yardstick against which results with other vascularized flaps (specifically muscle flaps) could be compared.

Methods: A retrospective review was undertaken of 85 consecutive patients in whom omentum was used in the chest. In 47 patients (group I), use of omentum was prophylactic to aid in the healing of closures or anastomoses considered to be at high risk for failure. In 32 patients (group II), omentum was used in the treatment of problems complicated by established infection. In 6 patients (group III), omentum was used for coverage of prosthetic chest wall replacements after extensive chest wall resection.

Results: Overall, omental transposition was successful in its prophylactic or therapeutic purpose in $88 \%$ of these difficult cases $(75 / 85)$. Success with omentum was achieved for $89 \%$ of patients (42/47) in group I, 91\% of patients (29/32) in group II, and $67 \%$ of patients (4/6) in group III. Three patients (3.5\%) had complications of omental mobilization. Four patients $(4.7 \%)$ died after the operation as a result of failure of the omentum to manage the problem for which it was used.

Conclusions: Results with omental transposition compare favorably with published series of similarly challenging cases managed with muscle transposition. Complications of omental mobilization are rare. We believe that its unique properties render the omentum an excellent choice of vascularized pedicle in the management of the most complex cardiothoracic surgical problems.

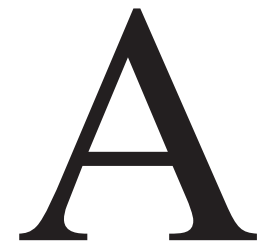

variety of vascularized tissue flaps have been used by cardiothoracic surgeons to aid in healing anastomoses or closures considered to be at risk for dehiscence and to help eradicate and sterilize infected spaces. Ischemia resulting from preoperative irradiation is the most common risk factor in cases of high-risk anastomoses. It is believed that reinforcing such anastomoses with a pedicle of well-vascularized tissue provides the oxygen and nutrients necessary for improved healing. Flaps for infected spaces can both obliterate the space and serve as a means of delivering antibiotics to the area. Most commonly used in each of these situations has been intrathoracic transposition of intercostal, serratus anterior, latissimus dorsi, or pectoralis major muscle. ${ }^{1}$ 
Although we too use muscle transpositions, our group has for many years found the omentum to be helpful in the most complex cases involving high-risk closures and infected spaces, as well as in a few cases of extensive chest wall resection where tissue coverage of prosthetic chest wall replacements is inadequate. The potential advantages of the omentum over muscle in these situations are several. In nearly all cases an omental pedicle that is based on the right gastroepiploic artery can reach any location in the chest. The omentum's bulk and pliability make it particularly well suited to completely filling irregular spaces and to closely adhering to at-risk anastomoses which may be in relatively inaccessible locations. Finally, in addition to bringing oxygenated blood to sites of ischemia, the omentum delivers vascular endothelial growth factor, a potent angiogenic factor. $^{2}$

We set out to review our experience with the omentum in the management of complex cardiothoracic problems during the last decade. We were particularly interested in determining (1) how results with omentum compared with published series of similar cases managed with other vascularized tissue flaps and (2) the frequency and severity of gastrointestinal complications of omental mobilization, which have been cited by some as a reason to favor chest wall muscle over omentum.

\section{Patients and Methods}

\section{Patients}

All patients operated on between 1987 and 1997 on the cardiac or thoracic surgical services at the Massachusetts General Hospital, Boston, who underwent omental transposition to the chest were included in this analysis. The hospital records of these 85 patients were reviewed carefully with respect to indication for surgery, operative procedure, postoperative course, outcome, and complications.

We found that patients could be grouped into three broad categories according to the indication for the use of omentum (Table 1). The first category (group I) consisted of 47 patients in whom the omentum was used not to treat an established complication but rather prophylactically to promote primary healing of bronchial or visceral closures or anastomoses that were considered to be at unusually high risk for failure. Twenty-six (55\%) of the 47 patients in group I had been irradiated (>4000 cGy), and $21(45 \%)$ were immunosuppressed (steroid at least equivalent to prednisone $5 \mathrm{mg}$ daily or recent chemotherapy). The operations performed in this group included 20 lung transplants, 14 airway resections, 7 cervical exenterations, and 6 pneumonectomies.

The second category (group II) consisted of 32 patients in whom the omentum was used to treat problems complicated by infection. Fourteen (44\%) of the 32 patients in group II had been irradiated, and $2(6.3 \%)$ were immunosuppressed. The conditions treated in this group included 15 bronchopleural fistulas (BPFs), 9 poststernotomy mediastinal infections, 3 esophageal perforations, and 5 miscellaneous other problems associated with infection.

The third and final category (group III) consisted of 6 patients who underwent extensive chest wall resections with omental cov-
TABLE 1. Results by category of patients undergoing omental transposition

\begin{tabular}{lrc}
\hline Category & No. & $\begin{array}{c}\text { Success } \\
\text { rate }\end{array}$ \\
\hline Group I (prophylaxis) & 47 & $89 \%$ \\
$\quad$ Lung transplantation & 20 & \\
$\quad$ Airway resection & 14 & \\
$\quad$ Carinal & 4 & \\
$\quad$ pneumonectomy & & \\
$\quad$ Tracheal resection and & 10 & \\
$\quad$ reconstruction & & \\
Cervical exenteration & 7 & \\
Pneumonectomy & 6 & \\
$\quad$ Completion & 2 & \\
$\quad$ Extrapleural & 1 & \\
Group II (infection) & 32 & \\
BPF & 15 & \\
$\quad$ Right & 11 & \\
$\quad$ Left & 4 & \\
Mediastinitis & 9 & \\
Esophageal perforation & 3 & \\
Miscellaneous & 5 & \\
Group III (chest wall & 6 & \\
resection) & & \\
Malignancy & & \\
Radiation ulcer & 4 & \\
Total & & \\
\hline$\quad$ & & \\
\hline
\end{tabular}

erage of prosthetic chest wall replacements. Five $(83 \%)$ of these patients had undergone previous irradiation.

Many of the patients in all three categories had undergone remote high-dose radiation. This is more likely to significantly compromise healing than is radiation administered immediately before an operation.

\section{Surgical Technique}

In each procedure the omentum was mobilized only to the extent necessary to reach the area of interest in the chest. The techniques of mobilization were as described previously. ${ }^{3}$ In some cases separation from the transverse colon was all that was required to allow sufficient mobility of the omentum to reach the area of interest. In most cases complete separation from the greater curve of the stomach as a pedicle that was based on the right gastroepiploic artery was required. In a few cases further elongation by dissection of the omental arcades was required. In these last cases, atraumatic vascular clamps were initially placed at the anticipated points of division of the arcades, and the distal portion of the omentum was examined for viability after approximately $10 \mathrm{~min}-$ utes. Only then did we divide the vessels that had been clamped.

Most commonly the omentum was mobilized through an upper midline abdominal incision and placed in a substernal location for later retrieval from within the chest after incision of the mediastinal pleura. The substernal route was also used to reach the neck. In some cases, notably single-lung transplantation, a subcostal incision was used for omental mobilization. In one case the omentum was mobilized through the thoracoabdominal incision performed for repair of an esophageal perforation. In 3 cases the 
omentum was passed through a defect created in the diaphragm rather than through a substernal tunnel. In each of these cases the omentum was tacked circumferentially to the diaphragmatic defect to prevent herniation of bowel. For chest wall resections, the omentum was brought through the upper part of the incision in the abdominal fascia and tunneled subcutaneously to the appropriate level on the thorax. In the cases of poststernotomy mediastinitis, the omentum was mobilized through an upper midline incision continuous with the sternotomy incision and brought directly into the mediastinum through the upper portion of the abdominal fascial incision. Whenever possible the abdominal incision was closed before thoracotomy or sternotomy in group II cases in which there was potential for spread of infection from the chest to the abdomen.

When the omentum was used to cover anastomoses or bronchial closures, it was tacked in circumferential fashion over the closure with multiple interrupted 4-0 silk or polyglycolic acid mattress sutures taken partial thickness through the bronchial wall, esophageal muscularis, or surrounding fibrous tissue. In this way the omentum was brought into intimate, direct contact with the entire area of interest. In cases in which there was sufficient length of omentum, a double layer of omentum was secured to the closure in similar fashion. In lung transplantation cases, the omentum was wrapped around the bronchial anastomosis and tacked circumferentially to the bronchus above and below the anastomosis. For bilateral lung transplantation the omentum was split according to its vascular supply into two lappets, each of which was tacked around one of the anastomoses.

In 7 of the cases the omental flap was combined with muscle flaps when complete coverage or filling of the ischemic or infected area could not be satisfactorily accomplished with omentum alone.

\section{Results}

\section{Group I: Prophylaxis for At-risk Anastomoses or Closures}

Overall, success in this group was achieved in 42 of 47 patients $(89 \%)$.

Lung transplants. Twenty patients underwent lung transplantation with wrapping of the anastomoses with omentum. Until 1993 this was part of our standard technique for lung transplantation. There were 17 single lung transplants (12 right and 5 left) and 3 bilateral sequential lung transplants.

Of the 20 patients, 19 had healing of their bronchial anastomoses without problems. There were no stenoses requiring dilation or stenting. One patient who had cystic fibrosis and underwent bilateral transplantation had separation of an anastomosis. This injury was granulating at the time of the patient's death from pneumonia during the initial hospitalization. There was one other in-hospital death in the group of patients in whom omentum was used; the cause was respiratory failure unrelated to any anastomotic problem.

There was one patient before 1993 who, because of a previous gastrectomy, had a pericardial fat pad rather than omentum used to wrap his anastomosis. This patient had stenosis of the anastomotic site, ultimately resulting in death from pneumonia. In the period after we had stopped using omentum routinely to wrap transplant anastomoses, there were 3 major anastomotic problems in 46 cases.

Airway resections. Fourteen patients underwent highrisk airway resections prophylactically treated with omental wrapping of the anastomosis. The tracheal resections among these were considered to pose higher risk than the usual because of either previous irradiation, long length of resection with resulting anastomotic tension despite release procedures, or partial devascularization as a result of limited lymph node dissection for tracheal malignancy.

Among these 14 patients, primary healing without complication was achieved in 12. The anastomosis failed in 2 patients. In 1 this resulted in a tracheoinnominate fistula and death. In the other a T-tube was required after anastomotic separation. Both of these patients had undergone remote, high-dose irradiation of the surgical field for unrelated malignancies. One had also undergone previous bilateral neck dissections.

Cervical exenterations. Seven patients underwent cervical exenteration and had omentum used to wrap the tracheal stoma and buttress the gastropharyngeal anastomosis. Wrapping the stoma in these cases was intended to guard against erosion into the innominate artery in the event of stomal dehiscence. ${ }^{4}$ In none of these cases were there either stomal or anastomotic complications, and all patients survived.

Pneumonectomies. Six patients underwent pneumonectomy with omental coverage. The risk factors included previous irradiation in 4 cases, immunosuppression in 3 cases, and ongoing infection in 3 cases. The bronchial stump was hand sewn with interrupted 4-0 polyglycolic acid suture in all cases.

Four of these 6 patients had uncomplicated healing of the bronchial stump. The first failure occurred in a woman who underwent salvage right pneumonectomy for a lung abscess eroding the right main bronchus that had developed during chemoradiotherapy for bronchogenic carcinoma. She died 5 weeks after the operation of respiratory failure with a BPF. The second failure occurred in a man who had a BPF develop after a right upper lobectomy with lateral bronchoplasty performed at another institution. We performed a sleeve resection, which also dehisced. The patient then underwent completion pneumonectomy with omental coverage; another BPF developed, from which he ultimately recovered.

\section{Group II: Problems Complicated by Infection}

Overall, success in this group was achieved in 29 of 32 cases $(91 \%)$.

Postpneumonectomy BPF. Fifteen patients with BPF were treated by omental transposition and coverage of a reclosed bronchial stump. The omentum in these cases 
served to both reinforce the bronchial closure and fill the empyema cavity. 5 All but 1 of the BPFs resulted from pneumonectomy performed at another institution. Six patients underwent an open drainage procedure (rather than tube thoracostomy) before the definitive operation for BPF closure. Eight patients underwent primary closure of the chest at the time of BPF repair, and 6 underwent a Clagett procedure some time after the BPF repair. In 3 cases muscle flaps were used to supplement the omentum.

Successful repair of the BPF was achieved in 13 of these 15 patients. One failure occurred in a patient who underwent only omental coverage of the BPF, without our usual suture closure of the defect. This was necessary because it was considered that the procedure should be expeditiously completed after an intraoperative cardiac arrest. The second failure in this group occurred in a patient who had development of a BPF with fungal empyema after bilobectomy. This patient ultimately underwent open window thoracostomy.

Poststernotomy mediastinal infections. Nine patients were treated with omental transposition for poststernotomy mediastinitis. Cardiac operations represented included isolated valve replacement, isolated coronary artery bypass, combined valve and coronary procedures, and aortic procedures. Before omental transposition, these patients had undergone a mean of 0.66 previous mediastinal débridements (range 0-3 débridements). Of the 9 patients in this group, 6 had infection of the bone and connective tissue alone, whereas 3 had associated infections of adjacent major vascular structures or prosthetic replacements of these vascular structures.

Successful control of the local infection was achieved with omental transfer in 8 of the 9 cases, although 2 patients died of indirectly related causes during the hospitalization. Of the 6 patients without major vascular structures infected, 5 were cured by omental transfer; the other had omental necrosis with recurrent infection, which was ultimately cured by rectus flap coverage and closure. Of the 3 patients with associated infection of surrounding vascular structures, 1 was cured, 1 died of adult respiratory distress syndrome without evidence of ongoing mediastinal infection, and 1 died of intra-abdominal sepsis without evidence of ongoing mediastinal infection. None of these patients, each of whom was seen for acute, massive bleeding from the ascending aorta or an ascending aortic graft as a result of eroding infection, had recurrent bleeding.

Esophageal perforations. Three patients with esophageal perforation were treated successfully with suture closure and omental buttressing. These patients included 1 with a Boerhaave perforation whose initial repair with an intercostal muscle flap failed, 1 with a leak after laparoscopic Nissen fundoplication, and 1 with an esophagocutaneous fistula after radiation therapy and laryngectomy for carcinoma of the thyroid.

Miscellaneous problems with infection. This complex and diverse group of 5 patients who did not fall within any of the previously categories were all treated successfully with omental transfer. The patients included 1 with a bronchoesophageal fistula after laser treatment of a histoplasmosis-related broncholith, 1 with a tracheoesophageal fistula after previous right upper lobectomy for cavitary infection, 1 with innominate artery hemorrhage as a result of erosion of an infected, radiation-induced skin ulcer, 1 with a disrupted tracheal anastomosis after tracheal resection of a subglottic cancer that also required lateral resection of the esophageal wall, and 1 with dehiscence of the left bronchial anastomosis after bilateral sequential lung transplantation in which pericardial fat had been used at the initial procedure.

\section{Group III: Extensive Chest Wall Resection}

In this group of 6 patients, complete healing of the chest wall defect was achieved in 4 (67\%) with omental coverage. The extent of resection included the entire sternum in 1 case, approximately $50 \%$ of the sternum in 3 cases, and segments of no less than four ribs in all but 1 case. Marlex mesh (Phillips Petroleum Company, Bartlesville, Okla) alone or in a Marlex-methylmethacrylate sandwich was used in all but 1 case. All patients had undergone irradiation, and 3 were immunosuppressed.

The 2 failures were as follows. One occurred in a man in whom primary closure was initially attempted after resection for malignant fibrous histiocytoma of the chest wall after preoperative radiation therapy. When this closure resulted in wound infection and separation, the wound was treated open until clean, and the patient then underwent omental coverage with simultaneous split-thickness skin grafting of the area. The omentum necrosed, presumably as a result of kinking of its vascular supply, resulting in loss of the graft and necessitating débridement. The wound was subsequently treated with dressing changes; it gradually closed but remained incompletely healed. The second failure in this group occurred in a woman who underwent resection of half of the sternum with parts of five ribs and Marlex mesh reconstruction for a radiation-induced ulcer after right mastectomy for breast carcinoma. Her large prosthetic procedure required both omentum and a latissimus dorsi muscle flap for complete coverage. Infection of the Marlex mesh developed, and the mesh was removed on postoperative day 30 , at which time the omentum and muscle remained healthy. The wound was subsequently treated open and allowed to granulate. It required one further operative débridement but subsequently healed down to a single draining sinus tract. 
TABLE 2. Mortality and omentum-related morbidity

\begin{tabular}{lcc}
\hline & No. & $\%$ \\
\hline Overall mortality & 7 & 8.2 \\
Mortality from omental failure & 4 & 4.9 \\
Ileus & 2 & 2.4 \\
Abdominal fascial dehiscence & 1 & 1.2 \\
Flap necrosis & 2 & 2.4 \\
\hline
\end{tabular}

\section{Complications of Omental Harvest}

Three patients (3.5\%) had gastrointestinal complications of omental harvest (Table 2). These included 2 cases of ileus and 1 of dehiscence of the abdominal fascial closure. The wound dehiscence was managed with early repair, and this patient had no further morbidity. One of the cases of ileus was mild and resolved with conservative management. The second case of ileus occurred in a critically ill patient with multiorgan system failure and was probably multifactorial in origin. The ileus was severe and persistent and required placement of a cecostomy tube. The patient subsequently had leakage of stool into his abdominal cavity at the site of the cecostomy, and he ultimately died of intra-abdominal sepsis. One patient (1.3\%) had partial necrosis of the omental flap that was believed to be due to compromise of its vascular supply (see group III).

Four patients (4.9\%) died after the operation as a direct result of failure of the omentum to manage the problem for which it was used. Their case histories are all described elsewhere in this section. Overall, 7 patients $(8.2 \%)$ died within the hospitalization during which the omental transposition was performed.

\section{Discussion}

The findings of this case series confirm that the omentum, which has been used extensively in the management of a variety of intra-abdominal conditions, is similarly effective in cardiothoracic cases characterized by high-risk anastomoses or closures (particularly after remote high-dose irradiation) or complicated by the presence of infection. Furthermore, we have found the rate of complications related to omental harvest to be low.

In light of the complexity of the cases reviewed here, we are pleased to report a success rate of $88 \%$. Combining this series with a previous series from our institution in which the omentum was used successfully for 35 of 37 patients with similar problems, ${ }^{3}$ our overall success with omentum in the management of complex cardiothoracic problems reaches $90 \%$. These results with omentum compare favorably with published series of similarly challenging cases managed with other vascularized tissues.

The Mayo Clinic reported 100 comparable patients managed with transposition of one or more muscles to an intrathoracic position (Table 3). ${ }^{1}$ This report, to our knowl-
TABLE 3. Results of current series with omentum versus Mayo Clinic series ${ }^{1}$ with muscle flaps

\begin{tabular}{lcc}
\hline Category & $\begin{array}{c}\text { Success rate (\%) with } \\
\text { omentum }(\mathbf{n}=\mathbf{7 9 *})\end{array}$ & $\begin{array}{c}\text { Success rate (\%) with } \\
\text { muscle }(\mathbf{n}=\mathbf{1 0 0})\end{array}$ \\
\hline Group I (prophylaxis) & 89 & 81.5 \\
Group II (infection) & 91 & 69.9 \\
Overall & 90 & 75 \\
Mortality & 8.2 & 16
\end{tabular}

*Patients undergoing chest wall reconstruction were excluded because such patients were not present in the comparable Mayo Clinic series.

edge the largest published series of intrathoracic muscle flaps, consisted of 27 patients in whom flaps were used prophylactically (analogous to our group I) and 73 patients in whom they were used to manage the complications of infection, including BPFs, empyemas, and esophageal fistulas (analogous to our group II). There were 16 operative deaths (16\%) in the Mayo Clinic series, as opposed to 8.2\% in our series. Sixty-nine percent of patients in the Mayo Clinic series were successfully treated with the first flap operation, versus $88 \%$ in our series. Parsing the results further, one finds that we achieved success in $91 \%$ of infected patients (group II), versus the Mayo Clinic's $69.9 \%$, and in $89 \%$ of prophylactically treated patients (group I), versus the Mayo Clinic's $81.5 \%$. If one were to exclude the transplant group from our group I analysis, because the Mayo Clinic series did not include transplants, one would still find an $85 \%$ success rate in prophylactically treated patients. In another report from the Mayo group consisting exclusively of BPFs, cure was achieved with muscle transposition followed by Clagett procedure in only $57 \%$ of 45 patients, ${ }^{6}$ versus $87 \%$ of 15 patients with BPF treated by omental transposition in our series.

It might reasonably be argued that it is impossible to know whether these retrospective reviews contain patients who are strictly comparable. Further, because the studies report on largely nonoverlapping time periods, with many improvements in operative, anesthetic, and postoperative management during the interval, any comparison must certainly be interpreted with caution. It must be noted, however, that because of the positive experience with omentum at the Massachusetts General Hospital it has been our policy to use omentum in the cases carrying the highest risks. We have used muscle flaps for patients ( 25 between 1987 and 1997) considered to be likely to respond to a flap that has been considered less effective but somewhat easier to harvest. For this reason, any selection bias in our series would tend to worsen our results with omental transposition.

A previous series of BPFs treated at our institution with either muscle $(n=14)$ or omental $(n=25)$ flaps allowed a more direct comparison of the results obtained with these two tissues than can be obtained by comparing retrospective 
studies from different institutions. ${ }^{5}$ In this group of patients, repair of the fistula was successful in 23 of 25 patients treated with omentum $(92 \%)$ and in 9 of 14 patients treated with muscle $(64 \%)$. It should be noted, further, that as a result of the bulk of the omentum, complete obliteration of the cavity and primary closure of the chest is frequently possible after omental transposition alone, as was the case in 8 of 15 patients reported on in this series (53\%). Although multiple muscle flaps may allow primary closure, as has been described by Miller and colleagues, ${ }^{7}$ this has not been the most frequently applied approach. The more commonly used regimen of open management after muscle transposition, followed by a delayed Clagett procedure, is costly, painful, and time-consuming.

Why might the omentum be a more effective tissue flap than muscle? One reason may be that whereas chest wall muscles frequently fall within the radiation ports that have been used to treat many of these patients, the omentum lies well outside of these ports. Thus muscles transposed into the chest may be compromised by the same radiation-induced ischemia that affects the region of intrathoracic pathologic damage. This is not an insignificant issue, because 53\% of patients in our series had undergone irradiation.

Second, in addition to bringing blood carrying oxygen and antibiotics to the area of interest, omentum has the additional benefit of delivering a potent angiogenic factor, ${ }^{8}$ which has recently been identified as vascular endothelial growth factor. ${ }^{2}$ This substance appears to be responsible for the demonstrated ability of the omentum to accelerate neovascularization across bronchial suture lines ${ }^{9}$ and to reduce the frequency of bronchial anastomotic complications in canine lung transplantation. ${ }^{10,11}$ No such factor is present in muscle. ${ }^{12}$

Finally, the amorphous shape and typical bulk of the omentum provide it with an advantage over muscle flaps. Its bulk serves, certainly more effectively than with single muscle flaps, to eliminate the dead space so often present in postpneumonectomy empyemas or poststernotomy mediastinal infections. The pliability of the omentum allows it to be intimately apposed to at-risk anastomoses or closures and to fill even the most irregular cavities.

With regard to chest wall defects, as can be surmised by the small number of cases accumulated, we use the omentum only in special situations. The primary indication has been an anterior defect of such great size that a rigid chest wall replacement, such as a Marlex methyl methacrylate sandwich, is indicated to preserve contour and pulmonary function. Although others question the need for rigid chest wall replacements, ${ }^{13}$ we favor them in certain cases, and it is our opinion that prosthetics of this size require excellent soft-tissue coverage to avert catastrophic infection. These defects are frequently so large that muscle flaps will not easily cover them, and we had considered the omentum more likely to prevent infection and provide a better bed for any skin grafting that might be required. The fact that the omentum failed in $33 \%$ of these cases of chest wall resection, however, suggests that for this use muscle flaps or myocutaneous flaps may well be better than omentum when they can provide sufficient coverage.

In addition to demonstrating the effectiveness of omentum, this series highlights the fact that there have been few complications related to the preparation of a vascularized omental pedicle and its translocation into the chest. None of our patients had herniation of abdominal organs into the chest either through a diaphragmatic defect created for passage of the omentum or through the substernal route. There were no instances of spread of intrathoracic infection to the abdomen. There were, in fact, no abdominal wound infections.

Of the 3 patients who had gastrointestinal complications, 2 had problems that were easily managed. The 1 patient who died of complications related to placement of a cecostomy tube for severe, persistent ileus was critically ill and in multiorgan system failure even before the onset of his ileus. This problem, then, was likely multifactorial and cannot be clearly attributed to the use of an omental flap. Stamatis and associates ${ }^{14}$ addressed the question of impaired gastrointestinal function after omental flap creation by performing a nuclear "milk scan" to evaluate gastric emptying after the procedure. They found no evidence of delayed gastric emptying in any of the 12 patients studied.

Two patients had failure of the omental flap as a result of necrosis of the pedicle. This was thought to result from rotation and kinking of the right gastroepiploic vessel in the single failure in the poststernotomy mediastinitis group. It was thought to result from kinking of the vessel at the site of its crossing the costal margin in the patient from the chest wall resection group whose flap necrosed. Such instances of tissue loss related to vascular occlusion may certainly occur after intrathoracic transposition of chest wall muscles as well, and there are no data to suggest that they are more likely to occur with omentum than with muscle. The occasional occurrence of this complication, however, highlights the importance of attention to detail during the performance of these operations.

In summary, this series of cases demonstrates that pedicled omentum is remarkably successful in the management of cardiothoracic surgical problems characterized by highrisk anastomoses or closures or by ongoing infection. Our limited data on the omentum in coverage of chest wall defects does not support its use for this indication. Aside from chest wall coverage, comparison of published series suggests that omentum is at least as effective as muscle transposition in the management of these difficult problems. In addition, we have found that the frequently cited risks of using omentum - that is, diaphragmatic hernia, gastrointes- 
tinal complications, and spreading of intrathoracic infection to the abdomen-occur only very rarely. We suggest that the omentum should be used more frequently in the management of the most complex cases-those complicated by previous irradiation, immunosuppression, devascularization, anastomotic tension, or infection.

\section{References}

1. Arnold PG, Pairolero PC. Intrathoracic muscle flaps: an account of their use in the management of 100 consecutive patients. Ann Surg. 1990;211:656-60.

2. Zhang QX, Magovern CJ, Mack CA, Budenbender KT, Ko W, Rosengart TK. Vascular endothelial growth factor is the major angiogenic factor in omentum: mechanism of omentum-mediated angiogenesis. J Surg Res. 1997;67:147-54.

3. Mathisen DJ, Grillo HC, Vlahakes GJ, Daggett WM. The omentum in the management of complicated cardiothoracic problems. J Thorac Cardiovasc Surg. 1988;95:677-84.

4. Grillo HC, Mathisen DJ. Cervical exenteration. Ann Thorac Surg. 1990;49:401-9.

5. Puskas JD, Mathisen DJ, Grillo HC, Wain JC, Wright CD, Moncure AC. Treatment strategies for bronchopleural fistula. J Thorac Cardiovasc Surg. 1995;109:989-96.
6. Pairolero PC, Arnold PG, Trastek VF, Meland NB, Kay PP. Postpneumonectomy empyema: the role of intrathoracic muscle transposition. J Thorac Cardiovasc Surg. 1990;99:958-68.

7. Miller J, Mansour K, Nahai F, Jurkiewicz M, Hatcher CJ. Single-stage complete muscle flap closure of the postpneumonectomy empyema space: a new method and possible solution to a disturbing complication. Ann Thorac Surg. 1984;38:227-31.

8. Goldsmith HS, Griffith AL, Kupferman A, Catsimpoolas N. Lipid angiogenic factor from omentum. JAMA. 1984;252:2034-6.

9. Morgan E, Lima O, Goldberg M, Ferdman A, Luk S, Cooper J. Successful revascularization of totally ischemic bronchial autografts with omental pedicle flaps in dogs. J Thorac Cardiovasc Surg. 1982; 84:204-10.

10. Lima O, Goldberg M, Peters W, Ayabe H, Townsend E, Cooper J. Bronchial omentopexy in canine lung transplantation. J Thorac Cardiovasc Surg. 1982;83:418-21.

11. Dubois P, Choiniere L, Cooper J. Bronchial omentopexy in canine lung allotransplantation. Ann Thorac Surg. 1984;38:211-4.

12. Silverman KJ, Lund DP, Zetter BR, Lainey LL, Shahood JA, Freiman DG, et al. Angiogenic activity of adipose tissue. Biomed Biophys Res Commun. 1988;153:347-52.

13. Arnold G, Pairolero PC. Chest wall reconstruction: an account of 500 consecutive patients. Plast Reconstr Surg. 1996;98:804-10.

14. Stamatis G, Freitag L, Wencker M, Greschuchna D. Omentopexy and muscle transposition: two alternative methods in the treatment of pleural empyema and mediastinitis. Thorac Cardiovasc Surg. 1994; 42:225-32.

\section{Availability of Journal back issues}

As a service to our subscribers, copies of back issues of The Journal of Thoracic and Cardiovascular Surgery for the preceding 5 years are maintained and are available for purchase from Mosby until inventory is depleted. Please write to Mosby, Subscription Customer Service, 6277 Sea Harbor Dr, Orlando, FL 32877, or call $800-654-2452$ or $407-345-4000$ for information on availability of particular issues and prices. 